\title{
GWAS and drug targets
}

\author{
Chen Cao ${ }^{1,2}$, John Moult ${ }^{1,3^{*}}$
}

From SNP-SIG 2013: Identification and annotation of genetic variants in the context of structure, function, and disease

Berlin, Germany. 19 July 2013

\begin{abstract}
Background: Genome wide association studies (GWAS) have revealed a large number of links between genome variation and complex disease. Among other benefits, it is expected that these insights will lead to new therapeutic strategies, particularly the identification of new drug targets. In this paper, we evaluate the power of GWAS studies to find drug targets by examining how many existing drug targets have been directly 'rediscovered' by this technique, and the extent to which GWAS results may be leveraged by network information to discover known and new drug targets.
\end{abstract}

Results: We find that only a very small fraction of drug targets are directly detected in the relevant GWAS studies. We investigate two possible explanations for this observation. First, we find evidence of negative selection acting on drug target genes as a consequence of strong coupling with the disease phenotype, so reducing the incidence of SNPs linked to the disease. Second, we find that GWAS genes are substantially longer on average than drug targets and than all genes, suggesting there is a length related bias in GWAS results. In spite of the low direct relationship between drug targets and GWAS reported genes, we found these two sets of genes are closely coupled in the human protein network. As a consequence, machine-learning methods are able to recover known drug targets based on network context and the set of GWAS reported genes for the same disease. We show the approach is potentially useful for identifying drug repurposing opportunities.

Conclusions: Although GWA studies do not directly identify most existing drug targets, there are several reasons to expect that new targets will nevertheless be discovered using these data. Initial results on drug repurposing studies using network analysis are encouraging and suggest directions for future development.

\section{Introduction}

Until recently, information on which variants within the human genome contribute to increased risk of common human disease was fragmentary and often statistically weak. New chip-based technologies and large-scale sequencing have now provided relatively unbiased and reliable information on SNVs (single nucleotide variants) and indels that are significantly associated with altered risk for a number of common diseases. To date, most information has been obtained through genome wide association studies (GWAS) using microarray technology, providing information only on common SNVs (the

\footnotetext{
* Correspondence: jmoult@umd.edu

'Institute for Bioscience and Biotechnology Research University of Maryland 9600 Gudelsky Drive, Rockville, MD 20850, USA

Full list of author information is available at the end of the article

single nucleotide polymorphisms, SNPs). The current generation of GWA studies typically include several thousand individuals with the disease of interest and a similar number of control individuals without the disease. These studies and meta-analyses combining data from multiple studies have now found more than 1600 loci where variants are associated with complex traits, including many diseases (the GWAS catalog, http:// www.genome.gov/gwastudies).

There have been a number of discussions on the efficacy of GWA studies [1]. In spite of the success in discovering disease associations, it is becoming clear that many disease mechanism genes with the highest effect on disease phenotypes are not discovered by GWAS. Studies of blood pressure provide a striking example. There is a long history of identification of genes affecting blood 
pressure using non-genomic methods, and 30 genes discovered in this way have provided successful targets for treating hypertension [2]. But only a few of these candidate genes and no drug targets are discovered in large scale GWAS [3]. Further, mouse knockout data suggest that some of the missing genes have very large effect sizes, with blood pressure changes of $10 \mathrm{~s}$ of $\mathrm{mm}$ of $\mathrm{Hg}$ [4], whereas the largest changes associated with marker SNPs in GWAS studies are between about 0.5 and $1 \mathrm{~mm}$ of $\mathrm{Hg}$.

Known drug targets - genes that usually have a large effect size on the corresponding disease phenotype, and so should be found by GWAS - provide a means of investigating whether non-discovery of mechanism genes is a general phenomenon. Here, we compare a set of reported mechanism genes in the GWAS catalog (http://www.genome.gov/gwastudies [5], January 2012) with a corresponding set of known drug target genes (obtained from Drugbank [6], January 2012) for the same diseases. We find that the overlap of these two sets is very low. We also investigate two possible explanations for low overlap. Finally, we consider the relationship between GWAS genes and drug targets in the context of a protein functional interaction network, and develop a machine learning method to predict new drug targets using the relationship between GWAS genes and known drug targets.

\section{Results}

\section{Comparison of the GWAS catalog and Drugbank shows} GWAS only detects a very small fraction of existing drug targets

We examined the relationship between genes in the GWAS catalog [5] and drug target genes in Drugbank [6]. The GWAS catalog (http://www.genome.gov/gwastudies/) is a comprehensive collection of results from published GWAS studies on a wide variety of disease and other traits such as height. Drugbank [6] is a database that combines detailed drug (i.e. chemical, pharmacological and pharmaceutical) data with comprehensive drug target information (sequence, structure, and pathway). We compiled a list of disease related traits in the GWAS catalog and extracted the reported genes for each of them. The disease list includes a number of cancers, a variety of complex trait diseases, and disease predisposition traits such as obesity and hypertension. We then found the drugs used in treatment of each of these traits in Drugbank, and extracted the drug target genes for each drug. Thus, for each trait, we have a list of GWAS reported genes and a list of drug targets. For the 88 GWAS diseases that have drugs in Drugbank, there are on average 29.2 GWAS reported genes and 24.0 drug targets for 19.9 drugs (Table 1). There are a total 23 instances of GWAS genes that are also drug targets for the same disease. Three of these genes are each drug targets for two different diseases, so that only 20 of the 856 drug target genes have been discovered in GWA studies of the corresponding traits. This is slightly larger than the overlap of approximately 5 from a completely random model, but is a very low number considering that altered activity of most drug target genes will influence the disease phenotype.

\section{Possible data related reasons for low overlap}

One possible cause of lower overlap is that in Drugbank, some drug targets do not have a known mechanism and are probably 'predicted' targets based on sequence similarity to other verified drug targets $[7,8]$, and thus may be incorrect. We therefore compiled a list of verified drug targets, all of which have known drug action mechanisms documented in Drugbank. We find similar results with this set to those for the complete list of drug targets. For those 353 drug targets for 81 diseases with known mechanisms and with corresponding GWAS studies, only 12 are discovered by GWAS (Additional file 1). On average, in this set there are 30 GWAS reported genes and 11.2 verified drug targets for each of these 81 diseases. A second possible cause of low overlap is mis-assignment of mechanism genes in the GWAS catalog. Marker SNPs (those associated with a trait) found in a GWAS locus are usually in linkage disequilibrium with many other SNPs covering a number of genes, any of which in principle might be in disease mechanism. In some cases, the catalog assignments may be incorrect, and the true mechanism gene in a locus may in fact be a drug target. We investigated the effect of this factor by comparing drug target/GWAS overlap described above with that obtained including all genes in each locus as candidates, rather than just those reported as candidates in the GWAS catalog. For the 58 diseases with sufficient information in the catalog, linkage disequilibrium expansion from marker SNPs increased the set of candidate genes from the 1997 reported to 4035, about a factor of two. The number of GWAS genes that are also drug targets increased from 18 to 24 . This small increase is comparable with the increase of 3 that is expected from the random model. Thus, the number of GWAS/drug target matches missed as a consequence of misidentification of candidate genes appears very small. A third data related factor is coverage by the tag SNPs on the microarrays used in GWAS studies. If there is no tag SNP in linkage disequilibrium with the underlying variant involved in a disease mechanism, that contribution to the trait will not be detected. A study of 160 non-GWAS derived candidate genes for blood pressure concluded that only half were adequately covered with tag SNPs on a 500K array [3], suggesting this is a significant factor. But overall, data considerations do not qualitatively change the picture of very low GWAS gene/drug target overlap. 
Table 1 Overlap between GWAS reported genes and drug targets

\begin{tabular}{|c|c|c|c|c|c|}
\hline Disease & $\begin{array}{l}\text { Number of } \\
\text { Drugs }\end{array}$ & $\begin{array}{l}\text { GWAS reported } \\
\text { genes }\end{array}$ & $\begin{array}{l}\text { Number of drug } \\
\text { targets }\end{array}$ & $\begin{array}{l}\text { GWAS overlap, same } \\
\text { disease* }\end{array}$ & $\begin{array}{l}\text { GWAS overlap, all } \\
\text { diseases** }^{* *}\end{array}$ \\
\hline Acute lymphoblastic leukemia & 6 & 19 & 10 & 0 & 3 \\
\hline Age-related macular degeneration & 9 & 23 & 2 & 1 & 2 \\
\hline Allergic rhinitis & 69 & 11 & 20 & 0 & 5 \\
\hline Alzheimer's disease & 5 & 54 & 179 & 0 & 40 \\
\hline Amyotrophic lateral sclerosis & 3 & 26 & 2 & 0 & 1 \\
\hline Ankylosing spondylitis & 39 & 17 & 29 & 0 & 9 \\
\hline Arthritis & 168 & 7 & 112 & 0 & 35 \\
\hline Asthma & 102 & 43 & 52 & 1 & 19 \\
\hline Atopic dermatitis & 12 & 8 & 3 & 0 & 1 \\
\hline Atrial fibrillation & 45 & 7 & 25 & 0 & 14 \\
\hline Attention deficit hyperactivity disorder & 3 & 81 & 1 & 0 & 1 \\
\hline Autism & 3 & 6 & 10 & 0 & 5 \\
\hline Basal cell carcinoma & 6 & 8 & 9 & 0 & 2 \\
\hline Bipolar disorder/Schizophrenia & 93 & 215 & 110 & 1 & 32 \\
\hline Blood pressure/Hypertension & 351 & 100 & 114 & 3 & 35 \\
\hline Breast cancer & 84 & 42 & 43 & 1 & 13 \\
\hline Celiac disease & 3 & 74 & 1 & 0 & 0 \\
\hline Chronic kidney disease & 8 & 69 & 6 & 0 & 2 \\
\hline Chronic lymphocytic leukemia & 14 & 17 & 29 & 0 & 5 \\
\hline Chronic myeloid leukemia & 6 & 9 & 15 & 0 & 6 \\
\hline Chronic obstructive pulmonary disease & 14 & 18 & 7 & 0 & 2 \\
\hline Colorectal cancer & 8 & 14 & 16 & 0 & 6 \\
\hline Coronary heart disease & 6 & 84 & 5 & 0 & 3 \\
\hline Crohn's disease & 7 & 136 & 23 & 0 & 9 \\
\hline Cystic fibrosis & 8 & 7 & 11 & 0 & 5 \\
\hline Depression/Depressive disorder & 45 & 68 & 73 & 0 & 17 \\
\hline Diabetes & 46 & 205 & 59 & 4 & 21 \\
\hline Duodenal ulcer & 8 & 2 & 18 & 0 & 5 \\
\hline Emphysema & 10 & 5 & 17 & 0 & 5 \\
\hline Endometrial cancer & 1 & 2 & 2 & 0 & 0 \\
\hline Endometriosis & 5 & 4 & 7 & 0 & 3 \\
\hline End-stage renal disease & 2 & 2 & 8 & 0 & 3 \\
\hline Epilepsy & 18 & 1 & 53 & 0 & 10 \\
\hline Esophageal cancer & 1 & 18 & 2 & 0 & 1 \\
\hline Gallstones & 1 & 1 & 1 & 0 & 0 \\
\hline Gastric cancer & 2 & 3 & 1 & 0 & 0 \\
\hline Glaucoma & 24 & 13 & 31 & 0 & 6 \\
\hline Glioblastoma & 2 & 1 & 1 & 0 & 0 \\
\hline Heart failure & 51 & 16 & 65 & 0 & 27 \\
\hline HIV/AIDS & 54 & 62 & 53 & 1 & 9 \\
\hline Hodgkin's lymphoma & 8 & 7 & 31 & 0 & 7 \\
\hline Hypertriglyceridemia & 2 & 5 & 4 & 0 & 3 \\
\hline Hypothyroidism & 5 & 43 & 8 & 1 & 5 \\
\hline Inflammatory bowel disease & 2 & 18 & 8 & 0 & 4 \\
\hline Kawasaki disease & 1 & 20 & 11 & 1 & 5 \\
\hline Malaria & 17 & 3 & 17 & 0 & 4 \\
\hline Male infertility & 6 & 5 & 3 & 0 & 3 \\
\hline Melanoma & 9 & 20 & 6 & 0 & 0 \\
\hline Menopause age & 9 & 23 & 15 & 0 & 4 \\
\hline Migraine & 20 & 7 & 46 & 0 & 10 \\
\hline
\end{tabular}


Table 1 Overlap between GWAS reported genes and drug targets (Continued)

\begin{tabular}{|c|c|c|c|c|c|}
\hline Multiple myeloma & 7 & 3 & 10 & 0 & 3 \\
\hline Multiple sclerosis & 10 & 123 & 30 & 1 & 12 \\
\hline Myocardial infarction & 29 & 14 & 44 & 0 & 17 \\
\hline Narcolepsy & 2 & 4 & 6 & 0 & 1 \\
\hline Nephropathy/Nephrotic syndrome & 20 & 26 & 38 & 0 & 9 \\
\hline Neuroblastoma & 2 & 2 & 6 & 0 & 2 \\
\hline Non-small cell lung cancer & 5 & 7 & 10 & 0 & 1 \\
\hline Obesity & 4 & 40 & 11 & 0 & 4 \\
\hline Osteoarthritis & 26 & 3 & 46 & 0 & 10 \\
\hline Osteoporosis & 13 & 10 & 10 & 0 & 2 \\
\hline Ovarian cancer & 5 & 10 & 4 & 0 & 1 \\
\hline Paget's disease & 4 & 9 & 6 & 0 & 1 \\
\hline Pancreatic cancer & 2 & 29 & 11 & 0 & 4 \\
\hline Panic disorder & 6 & 10 & 18 & 0 & 4 \\
\hline Parkinson's disease & 20 & 62 & 184 & 1 & 34 \\
\hline Polycystic ovary syndrome & 2 & 7 & 2 & 0 & 1 \\
\hline Prostate cancer & 14 & 94 & 21 & 0 & 8 \\
\hline Psoriasis/Psoriatic arthritis & 19 & 30 & 39 & 0 & 13 \\
\hline Refractive error & 1 & 4 & 4 & 0 & 1 \\
\hline Restless legs syndrome & 2 & 6 & 18 & 0 & 6 \\
\hline Rheumatoid arthritis & 46 & 67 & 80 & 2 & 29 \\
\hline Sleepiness & 1 & 2 & 2 & 0 & 0 \\
\hline $\begin{array}{l}\text { Stevens-Johnson syndrome/toxic } \\
\text { epidermal necrolysis }\end{array}$ & 1 & 12 & 1 & 0 & 0 \\
\hline Stroke & 8 & 4 & 7 & 0 & 6 \\
\hline Tardive dyskinesia & 3 & 1 & 22 & 0 & 7 \\
\hline Testicular cancer & 4 & 7 & 6 & 0 & 2 \\
\hline Thyroid cancer & 2 & 5 & 3 & 0 & 2 \\
\hline Tuberculosis & 12 & 5 & 18 & 0 & 4 \\
\hline Type 1 diabetes & 8 & 74 & 18 & 0 & 8 \\
\hline Type 2 diabetes & 28 & 91 & 34 & 3 & 13 \\
\hline Ulcerative colitis & 5 & 95 & 9 & 1 & 6 \\
\hline Uterine fibroids & 1 & 7 & 1 & 0 & 0 \\
\hline Venous thromboembolism & 1 & 7 & 3 & 0 & 2 \\
\hline Vitiligo & 4 & 25 & 8 & 1 & 2 \\
\hline Mean & 19.90 & 29.18 & 24.00 & 0.26 & 7.09 \\
\hline
\end{tabular}

*For each disease, the number of GWAS reported genes that are also drug targets for the disease.

**For each disease, the number of GWAS reported genes that are drug targets for any disease.

Analysis using 1000 genomes data shows Drug Target genes have fewer high frequency non-synonymous SNPs than GWAS reported genes

We next consider two possible reasons why GWAS identifies so few known drug targets. A study of all the SNPs in the GWAS catalog [5] has shown that reported SNPs are common (median risk allele frequency $36 \%$, interquantile range (IQR) $21 \%-53 \%$ ), and are associated with modest effect size (median odds ratio 1.33 , IQR 1.20-1.61). We speculated that drug target genes may escape GWAS studies because these contain few common SNPs that affect function. To test this hypothesis, we examined the distribution of SNP frequencies and
SNP effect size in GWAS identified genes and drug targets, using SNP frequencies calculated from 1000 genomes data [9].

A SNP may affect in vivo function of a gene product through a number of different mechanisms, including modified protein function or protein stability, altered regulation of gene expression, modified splicing, and changed stability of messenger RNA. We focus on nonsynonymous SNPs, which have been shown to be significantly overrepresented at amongst GWAS marker SNPs [5]. We found that drug targets genes do have fewer non-synonymous SNPs (0.0155/residue vs. 0.0171/residue) and the tendency is more significant for common 
Table 2 Comparison of common non-synonymous SNP densities between GWAS reported genes and drug targets

\begin{tabular}{|c|c|c|c|c|}
\hline & Drug Targets & GWAS reported genes & HGMD genes & All genes \\
\hline Density of all non-synonymous SNPs & 0.0155 & 0.0171 & 0.0166 & 0.0171 \\
\hline Density of Common non-synonymous SNPs & $\begin{array}{r}0.00169 \\
P=0.0017^{1} \\
P=0.0023^{2}\end{array}$ & 0.00221 & 0.00179 & 0.00214 \\
\hline
\end{tabular}

${ }^{1} \mathrm{P}$-value for Mann-Whitney test against the density of common non-synonymous SNPs for GWAS reported genes. ${ }^{2} \mathrm{P}$-value for Mann-Whitney test against the density of common non-synonymous SNPs for all genes.

(Allele frequency $>5 \%$ ) non-synonymous SNPs $(0.00169 /$ residue vs. $0.00221 /$ residue, Mann-Whitney test $\mathrm{P}=0.0017$ ) (Table 2 ). We also included a set of predominantly monogenic disease genes from the Human Gene Mutation Database (HGMD) [10], expecting these to also be under negative selection pressure. SNP density is also lower in this class of genes. A possible explanation for the low occurrence of common SNPs is that the activity level of drug targets genes is strongly coupled to the disease phenotype. As a result they are under relatively high selection pressure, and SNPs with a substantial impact on function will be eliminated or tend to be at a low frequency.

Evolutionary analysis shows drug target genes are under slightly stronger negative selection than GWAS reported genes

If the drug targets genes are under stronger selection as we propose on the basis of SNP density, that effect should also be observable in the rate of sequence change during the evolutionary history of the gene family. The ratio of the rate of non-synonymous to synonymous change, $\mathrm{dN} / \mathrm{dS},[11]$ for a gene provides one measure to detect such selection pressure. We compared the $d N / d S$ for GWAS and drug target genes using human-mouse and human-chimp data from $\mathrm{H}$-invDB [12] and found both are under stronger selection (Table 3) than all genes. We found HGMD genes [10] also exhibit negative selection in recent history $(\mathrm{dN} / \mathrm{dS}$ calculated using human-chimp orthologs). The selection against variants in drug target genes is slightly stronger than that against variants in GWAS reported genes (Table 3) for $\mathrm{dN} / \mathrm{dS}$ calculated using human-chimp orthologs, suggesting the selection is stronger for drug targets in recent history.

The influence of transcript length

For some mechanisms, for example those arising from missense SNPs, the probability of contributing to a complex trait is dependent on the length of the gene affected: Under similar selection pressures, the longer the gene, the more likely variants affecting gene function will be present. Other mechanisms, such as those directly affecting transcription rate, are not length dependent. To test for a length effect, we examined the length distribution for GWAS reported genes, for drug targets, and for all genes (Figure 1). GWAS reported genes are significantly longer than the drug target genes (paired Mann-Whitney test, $\mathrm{P}=1.89 \mathrm{e}^{-6}$ ) and GWAS reported genes tend to be longer than all other genes. The mean longest transcript length for GWAS reported genes is about $110 \mathrm{~K}$ while the mean longest transcript length for drug targets is about $60 \mathrm{~K}$, almost a factor two different. The outlier here is the GWAS gene set - drug targets have a similar distribution to that of all genes.

Table $3 \mathrm{dN} / \mathrm{dS}$ analysis for GWAS reported genes and drug targets

\begin{tabular}{|c|c|c|c|c|c|}
\hline & & $\begin{array}{l}\text { Number of } \\
\text { genes }\end{array}$ & $\begin{array}{l}\text { Mean } \\
\text { dN/dS }\end{array}$ & $\begin{array}{l}\text { P Value for Mann-Whitney test } \\
\text { against all genes }\end{array}$ & $\begin{array}{l}\text { P Value for Mann-Whitney test against } \\
\text { GWAS reported genes }\end{array}$ \\
\hline \multirow{5}{*}{$\begin{array}{l}\text { Human-Mouse } \\
\text { orthologs }\end{array}$} & All genes & 13691 & 0.22 & & \\
\hline & GWAS reported genes & 2932 & 0.19 & $2.44 \mathrm{e}^{-09 *}$ & \\
\hline & Drug targets & 1035 & 0.18 & $1.21 e^{-04 *}$ & 0.43 \\
\hline & $\begin{array}{l}\text { Drug targets with } \\
\text { known mechanism }\end{array}$ & 432 & 0.17 & $6.04 e^{-06 *}$ & $0.038^{*}$ \\
\hline & HGMD genes & 720 & 0.20 & 1.0 & \\
\hline \multirow{5}{*}{$\begin{array}{l}\text { Human- } \\
\text { Chimpanzee } \\
\text { orthologs }\end{array}$} & All genes & 14173 & 0.44 & & \\
\hline & GWAS reported genes & 2911 & 0.36 & $1.26 \mathrm{e}^{-13 *}$ & \\
\hline & Drug targets & 1020 & 0.33 & $2.78 e^{-13 *}$ & $0.0098^{*}$ \\
\hline & $\begin{array}{l}\text { Drug targets with } \\
\text { known mechanism }\end{array}$ & 423 & 0.32 & $4.20 e^{-08 *}$ & $0.013^{*}$ \\
\hline & HGMD genes & 699 & 0.36 & $0.002^{*}$ & \\
\hline
\end{tabular}

\footnotetext{
"*” denotes significant, i.e, $\mathrm{P}<0.05$
} 


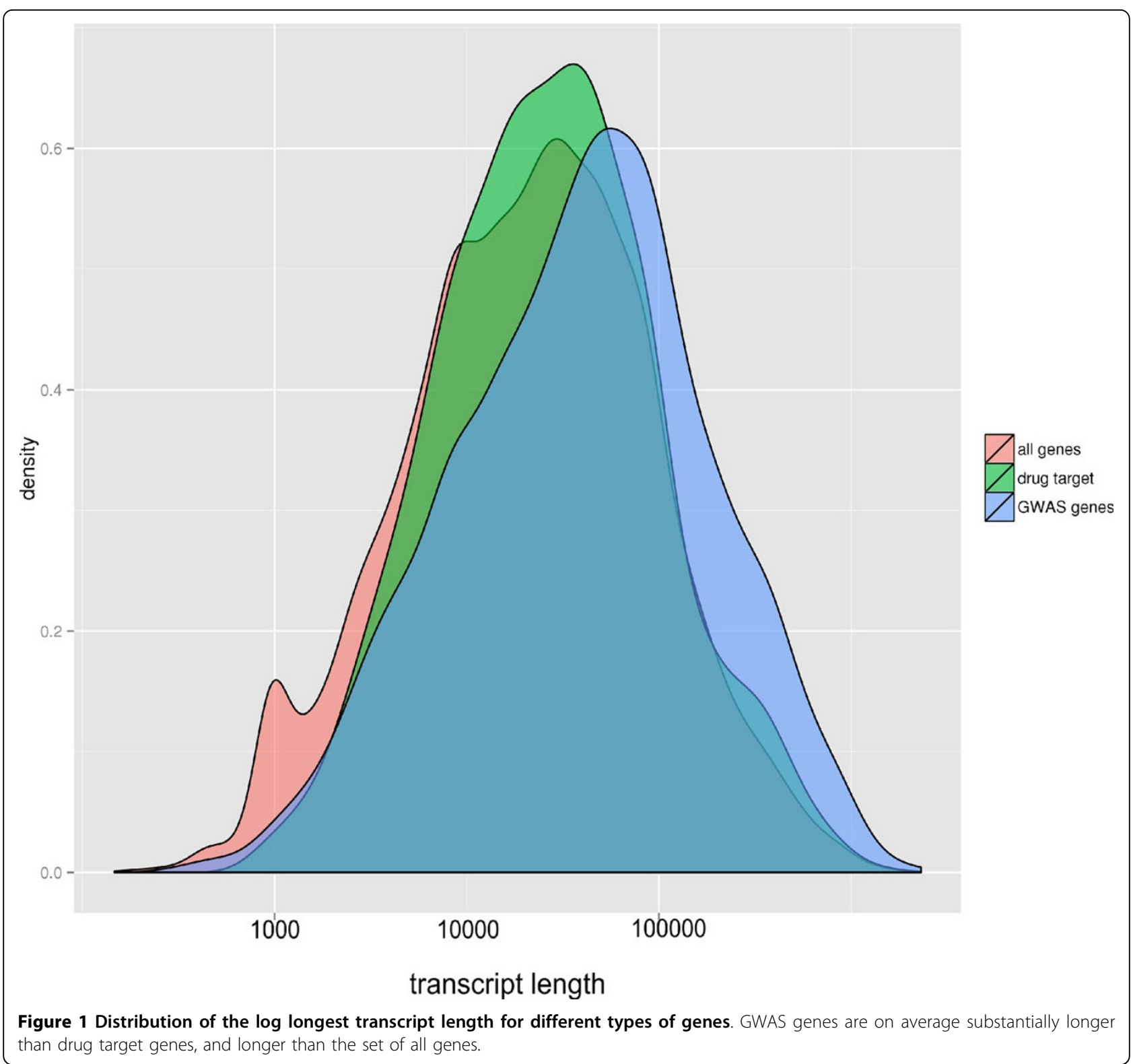

Thus there is a strong length factor influencing whether or not variants in a gene contribute to a complex trait. This result is consistent with a role for length dependent mechanisms, although there could be other explanations.

\section{Network analysis shows GWAS reported genes are close to drug target genes in a biological network}

Although most drug targets are not identified through GWAS studies, they are obviously as much involved in the disease mechanism as GWAS genes, and so may be expected to have similar properties, particularly in terms of pathway and network relationships. A number of studies have incorporated network information to aid in identifying various classes of genes, for example using a network module formalism to combine signals from multiple GWAS studies [13,14] and using network flow models to predict drug targets from expression and other data in prostate cancer [15]. Network models have also been used to identify pathways implicated in cancer [16]. It has already been observed that GWAS genes are substantially more closely connected in a functional network [17] than random genes, and we expect that to be the case for other large effect genes, such as known drug targets.

There are many resources available for different types of human biological networks. Protein-Protein interaction data $[18,19]$ have a wide coverage but usually have a high false positive rate. Curated pathways such as 
KEGG [20] and BioCarta (http://www.biocarta.com/ genes/index.asp) are considered to be more accurate but the coverage is sparse [21]. For our purposes, networks built from other kinds of relationship, such as regulatory networks deduced from micro-array data [22,23] or networks based on biochemical reactions [24] are too narrow in terms of the interactions they capture.

In this study, we use the Functional Interaction (FI) network from [21], a protein functional interaction network generated by extending curated biological pathways with non-curated sources of information, including protein-protein interactions, gene co-expression, protein domain interaction, Gene Ontology (GO) annotations and text-mined protein interactions, and covering about $50 \%$ of human genes. The network strikes a balance between experimentally validated results and prediction, with the prediction portion benchmarked by a reasonably rigorous process. We were able to map 611 out of 821 drug targets genes and 1125 out of 1914 GWAS reported genes for the 88 diseases to the network.

Examination of the network proximity of GWAS genes to each other and to drug targets for the same disease indeed shows a close-nit matrix of relationships. Figure 2 shows the network formed for the 43 GWAS and 16 drug target genes [6] for Type I Diabetes that project onto the FI network, and only including genes from these two sets which are linked by not more than one other intermediate gene. All drug targets and all but five of the GWAS genes form part of a single continuous sub-network. This suggests that the two sets of genes are indeed relatively close

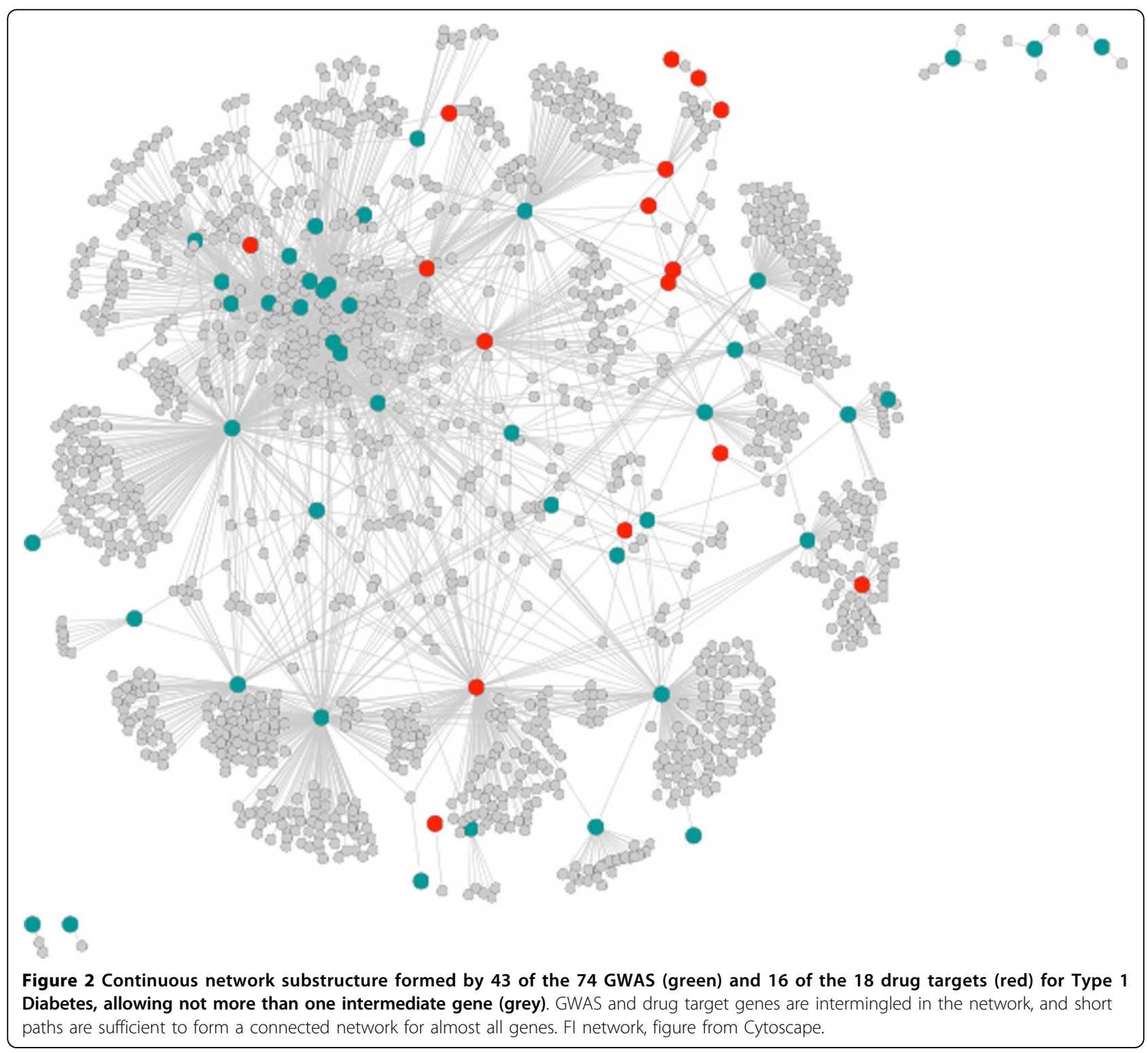


in their biological function. One measure of the relationship between GWAS reported genes and drug target genes is the closeness of each GWAS gene to its nearest drug target (Figure 3). The distributions show that distances from a GWAS reported gene to the closest drug target are on average much shorter than those of a random gene to a closest drug target, and the shortest distance from a drug target gene to the closest GWAS reported gene is also shorter than that of a random gene to the closest GWAS reported gene. Notably, drug targets are about

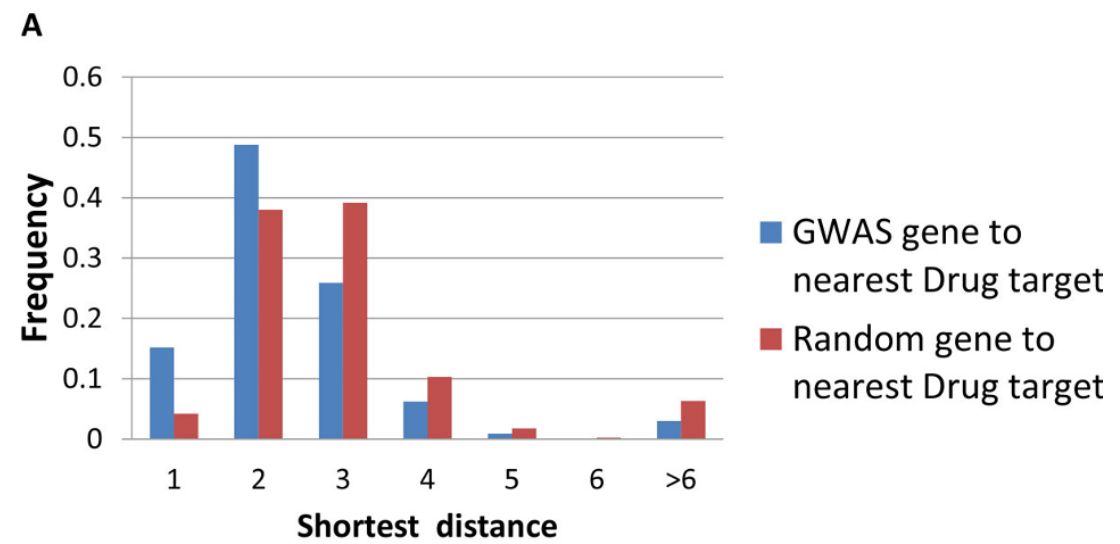

B

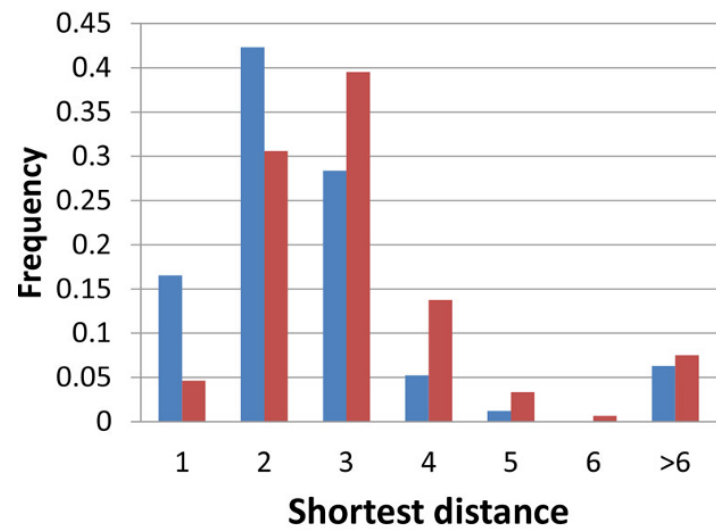

Drug target to nearest GWAS gene

All gene to nearest GWAS gene

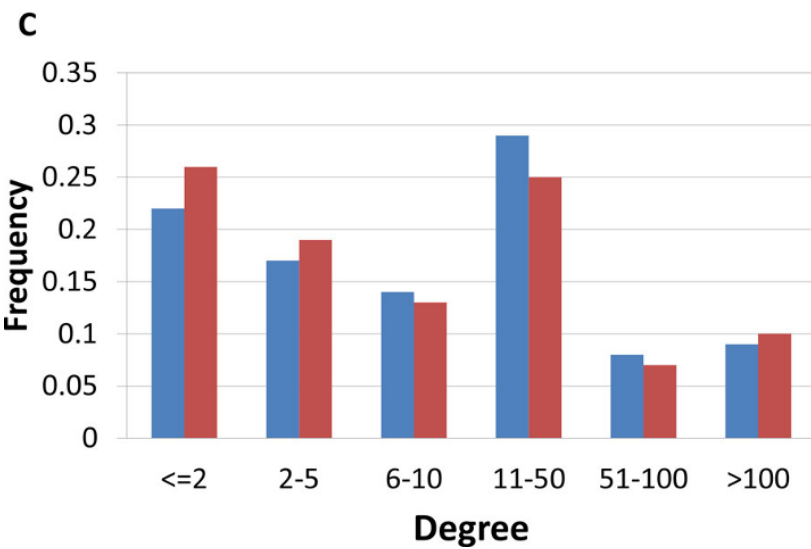

Drug Targets

All genes

Figure $3 \mathrm{~A}$. Distribution of shortest distances to the nearest drug target for GWAS reported genes and all genes. B. Distribution of the shortest distance to the nearest GWAS genes for drug targets and all genes. C. Distribution of degree for drug targets and all genes in the FI network. Drug targets have a slightly higher degree (Mann-Whitley test $P=0.014$ ). 
three fold enriched in the first neighbors of GWAS genes and are also enriched in GWAS second neighbors (genes two steps away in the gene network) (Figure 3).

Highly connected genes have more neighbors, and thus are more likely to include GWAS genes as neighbors. Thus, the observed enrichment of short paths between drug targets and GWAS genes could partially be a consequence of higher connectivity for drug targets. To control for this effect, we compared the degrees of drug targets with all genes (Figure 3) and found drug targets have a slightly higher degree (Mann-Whitney test, $\mathrm{P}=0.014$ ) on average. However the difference is marginal, and is unlikely to significantly contribute to the substantial difference between the short path distribution for drug targets and all genes.

\section{A machine learning method for drug target discovery}

The relationship between drug targets and GWAS genes revealed in the network analysis suggests that it should be possible to identify potential new drug targets from GWAS genes using machine learning methods trained on network features. The idea is to evaluate the probability that any gene is a potential drug target, given its network environment. The environment of each gene is represented by a set of features. Since we observed a threefold enrichment of drug targets in the first neighbors of the GWAS genes, we use the number of GWAS neighbors for a gene as a feature. This quantity is highly dependent on the total number of neighbors a gene has, so we also use the degree of the gene as a control. As the previous analysis shows, second neighbors of drug targets genes (genes that are two steps away in the protein interaction network) are also enriched for GWAS genes, thus we also use the number of second neighbor GWAS genes of a gene as a feature. These three features capture the enrichment information from the analysis above, but there are some subtle relationships not included. The problem of identifying drug targets based on their relationship to GWAS genes is similar to the problem of finding missing relationships in social network analysis. We therefore also use common friends with GWAS genes, a widely used feature in the social network machine learning field [25]. The common neighbor feature is defined as the proportion of neighbors shared by two genes:

$$
\text { Common Neighbor }(A, B)=\frac{\operatorname{count}\left(N_{A} \cap N_{B}\right)}{\operatorname{count}\left(N_{A} \cup N_{B}\right)}
$$

In which $\mathrm{N}_{A}$ is the set of Neighbors for gene $A, N_{B}$ is the set of Neighbors for gene $B$.

The total number of features for each gene is $3+\mathrm{N}$, where $\mathrm{N}$ is the number of GWAS genes for that disease that are mapped to the protein network. Since the number of drug targets (average 30) for a disease is very small compared to the total number of genes in the FI network (10956), the training set is highly unbalanced if we use the latter as the true negative set. To address this issue, we focus on the 932 existing drug targets in Drugbank that are also in the FI network, and thus restrict the task to identifying targets for existing drugs that can potentially be repurposed to treat other diseases. Repurposing is an attractive goal, since such use is much easier than developing a new drug from scratch [26].

We include the 30 diseases with at least 10 approved drug targets and 10 GWAS genes in the FI network. We tested four machine learning methods using the WEKA software package [27]: a SVM with a polynomial kernel, a SVM with a RBF kernel, a Naïve Bayes Network, and Random Forests. Among these the best result is achieved by a Random forest (Table 4). The best case is Kawasaki disease, with a true positive rate of $70 \%$ (recovering seven out of the 10 known drug targets) and a false positive rate of $2.7 \%$.

\section{Potential new drug targets for drug repurposing}

The 'false positive' drug targets are drug targets for other diseases which have very similar network properties to those of the disease under study. These may indeed be mistakes made by the classifier. However, some of these 'false positive' drug targets may be good candidates for repurposing, not previously identified.

For example, C1QB and C1QC are the highest scoring proteins in the false positive list for the best case, Kawasaki disease, These are subcomponents of complement C1Q. C1Q has been shown to be associated with lupus erythematous [28-30], another autoimmune disease related to Kawasaki disease [31,32], consistent with relevance to Kawasaki. C1Q is the target of several FDA approved drugs, for example, Etanercept, a drug treating rheumatoid arthritis and Adalimumab, a drug treating rheumatoid arthritis, psoriatic arthritis, ankylosing spondylitis, and other immune system mediated diseases. Thus these drugs may be potential candidates for use against Kawasaki disease.

Another disease where the method performs well is acute lymphoblastic leukemia (ALL), with a false positive rate of $7 \%$ and a true positive rate of $70 \%$. There is a relatively long list of 'false positive' targets (Table 5). Careful inspection of these genes reveals some that may have relevance to acute lymphoblastic leukemia, and so drugs for which these are targets provide potential candidates for repurposing. For example, chromosomal aberrations (i.e. chromosome translocation) in FGFR1 are associated with stem cell myeloproliferative disorder and stem cell leukemia lymphoma syndrome (provided by RefSeq, Jul 2008). FGFR1 is the drug target of Palifermin, a recombinant human keratinocyte growth factor (KGF) for the treatment of oral mucositis associated 
Table 4 Machine learning results for different diseases, using a Random Forest.

\begin{tabular}{|c|c|c|c|c|c|c|c|c|}
\hline Disease & $\begin{array}{l}\text { GWAS } \\
\text { genes }\end{array}$ & $\begin{array}{l}\text { Drug targets } \\
\text { (Mapped in } \\
\text { Network) }\end{array}$ & $\begin{array}{l}\text { True } \\
\text { Positive }\end{array}$ & $\begin{array}{l}\text { False } \\
\text { Positive }\end{array}$ & Precision & Recall & $\begin{array}{l}\text { ROC } \\
\text { area }\end{array}$ & $\begin{array}{l}\text { F- } \\
\text { Measure }\end{array}$ \\
\hline Ankylosing spondylitis & 17 & $29(24)$ & 0.36 & 0.123 & 0.074 & 0.36 & 0.73 & 0.123 \\
\hline Menopause & 24 & $15(14)$ & 0.571 & 0.098 & 0.082 & 0.571 & 0.819 & 0.143 \\
\hline Multiple sclerosis & 126 & $30(28)$ & 0.393 & 0.052 & 0.19 & 0.393 & 0.75 & 0.256 \\
\hline Myocardial infarction & 14 & $44(40)$ & 0.175 & 0.135 & 0.055 & 0.175 & 0.571 & 0.084 \\
\hline $\begin{array}{l}\text { Nephropathy/Nephrotic } \\
\text { syndrome }\end{array}$ & 26 & $38(35)$ & 0.371 & 0.245 & 0.056 & 0.371 & 0.576 & 0.097 \\
\hline Obesity & 40 & $11(11)$ & 0.273 & 0.098 & 0.032 & 0.273 & 0.724 & 0.058 \\
\hline Osteoporosis & 10 & $10(10)$ & 0.2 & 0.189 & 0.011 & 0.2 & 0.546 & 0.022 \\
\hline Pancreatic cancer & 29 & $11(6)$ & 0.167 & 0.1 & 0.011 & 0.167 & 0.611 & 0.02 \\
\hline Panic disorder & 10 & $18(16)$ & 0.438 & 0.118 & 0.061 & 0.438 & 0.754 & 0.107 \\
\hline Parkinson's disease & 62 & 184(132) & 0.606 & 0.226 & 0.307 & 0.606 & 0.712 & 0.407 \\
\hline Asthma & 43 & $52(47)$ & 0.213 & 0.102 & 0.1 & 0.213 & 0.713 & 0.136 \\
\hline Prostate cancer & 95 & $21(18)$ & 0.5 & 0.073 & 0.118 & 0.5 & 0.686 & 0.191 \\
\hline Psoriasis/Psoriatic arthritis & 31 & $39(36)$ & 0.5 & 0.076 & 0.209 & 0.5 & 0.852 & 0.295 \\
\hline Rheumatoid arthritis & 67 & $80(68)$ & 0.324 & 0.131 & 0.163 & 0.324 & 0.677 & 0.217 \\
\hline Type 1 diabetes & 76 & $18(16)$ & 0.25 & 0.104 & 0.04 & 0.25 & 0.631 & 0.07 \\
\hline Type 2 diabetes & 92 & $34(28)$ & 0.214 & 0.116 & 0.054 & 0.214 & 0.595 & 0.086 \\
\hline Bipolar disorder/Schizophrenia & 217 & $110(81)$ & 0.593 & 0.15 & 0.273 & 0.593 & 0.744 & 0.374 \\
\hline Blood pressure/Hypertension & 101 & 114(102) & 0.412 & 0.143 & 0.261 & 0.412 & 0.717 & 0.319 \\
\hline Breast cancer & 43 & $43(38)$ & 0.289 & 0.072 & 0.147 & 0.289 & 0.745 & 0.195 \\
\hline Chronic lymphocytic leukemia & 17 & $29(26)$ & 0.423 & 0.098 & 0.11 & 0.423 & 0.653 & 0.175 \\
\hline Colorectal cancer & 14 & $16(16)$ & 0.25 & 0.154 & 0.028 & 0.25 & 0.53 & 0.05 \\
\hline Acute lymphoblastic leukemia & 19 & $10(10)$ & 0.7 & 0.07 & 0.097 & 0.7 & 0.889 & 0.171 \\
\hline Crohn's disease & 139 & $23(22)$ & 0.455 & 0.093 & 0.105 & 0.455 & 0.764 & 0.171 \\
\hline Depression/Depressive disorder & 68 & $73(62)$ & 0.597 & 0.172 & 0.198 & 0.597 & 0.722 & 0.297 \\
\hline Diabetes & 209 & $59(51)$ & 0.216 & 0.081 & 0.134 & 0.216 & 0.712 & 0.165 \\
\hline Allergic rhinitis & 11 & 20(19) & 0.263 & 0.128 & 0.041 & 0.263 & 0.589 & 0.071 \\
\hline Glaucoma & 14 & $31(25)$ & 0.16 & 0.189 & 0.023 & 0.16 & 0.443 & 0.04 \\
\hline Alzheimer's disease & 54 & $179(125)$ & 0.544 & 0.178 & 0.321 & 0.544 & 0.69 & 0.404 \\
\hline Heart failure & 16 & $65(54)$ & 0.481 & 0.222 & 0.118 & 0.481 & 0.655 & 0.189 \\
\hline HIV/AIDS & 63 & $53(34)$ & 0.353 & 0.121 & 0.099 & 0.353 & 0.715 & 0.155 \\
\hline Kawasaki disease & 20 & $11(10)$ & 0.7 & 0.027 & 0.219 & 0.7 & 0.919 & 0.333 \\
\hline
\end{tabular}

with chemotherapy and radiation therapy. It's also the target for several experimental drugs.

A second potential repurposing target for acute lymphoblastic leukemia is the oncogene RET. Previous studies found differential expression of RET in acute myeloid leukemia [33], a distinct but related leukemia. In the version of Drugbank used in this analysis, there is no drug targeting RET for the treatment of ALL. Recently, however, the drug Ponatinib has been approved by the FDA for treatment of Philadelphia chromosome positive acute lymphoblastic leukemia $(\mathrm{Ph}+\mathrm{ALL})$ resistant or intolerant to prior tyrosine kinase inhibitor therapy. Thus, one of the high scoring ALL potential drug targets has now been approved for use for use with a new drug.

\section{Methods}

Connecting GWAS reported genes with drug targets using drug indication information from Drugbank

GWAS reported genes: The GWAS catalog was downloaded from http://www.genome.gov/admin/gwascatalog. txt in January 2012. Non-disease traits were removed by hand and multiple studies for each disease were combined into unique sets. 'Reported genes' were extracted to provide the list of GWAS genes for each disease.

Drug targets: Drugbank data were downloaded from http://www.drugbank.ca/downloads in January 2012. Drugs for each disease in the GWAS list were identified by searching the 'indication' information for all drugs in Drugbank. Then for each of these drugs, we extract all of the corresponding target genes. 
Table 5 Top 'false positive' drug targets for acute lymphoblastic leukemia.

\begin{tabular}{|c|c|c|}
\hline Target & Description from Refseq & $\begin{array}{l}\text { Random Forest } \\
\text { Probability }\end{array}$ \\
\hline MAPK3 & $\begin{array}{l}\text { The protein encoded by this gene is a member of the MAP kinase family. MAP kinases, also known as extracellular } \\
\text { signal-regulated kinases (ERKs), act in a signaling cascade that regulates various cellular processes such as } \\
\text { proliferation, differentiation, and cell cycle progression in response to a variety of extracellular signals. }\end{array}$ & 1 \\
\hline PIK3R1 & $\begin{array}{l}\text { Phosphatidylinositol 3-kinase plays an important role in the metabolic actions of insulin, and a mutation in this } \\
\text { gene has been associated with insulin resistance. }\end{array}$ & 0.96 \\
\hline RAF1 & v-raf-1 murine leukemia viral oncogene homolog 1 & 0.96 \\
\hline EGFR & $\begin{array}{l}\text { Mutations in this gene are associated with lung cancer. Multiple alternatively spliced transcript variants that encode } \\
\text { different protein isoforms have been found for this gene }\end{array}$ & 0.96 \\
\hline FGFR2 & $\begin{array}{l}\text { Mutations in this gene are associated with Crouzon syndrome, Pfeiffer syndrome, Craniosynostosis, Apert syndrome, } \\
\text { Jackson-Weiss syndrome, Beare-Stevenson cutis gyrata syndrome, Saethre-Chotzen syndrome, and syndromic } \\
\text { craniosynostosis. }\end{array}$ & 0.96 \\
\hline KDR & $\begin{array}{l}\text { This receptor, known as kinase insert domain receptor, is a type III receptor tyrosine kinase. Mutations of this gene } \\
\text { are implicated in infantile capillary hemangiomas. }\end{array}$ & 0.94 \\
\hline FLT1 & $\begin{array}{l}\text { This protein binds to VEGFR-A, VEGFR-B and placental growth factor and plays an important role in angiogenesis } \\
\text { and vasculogenesis. }\end{array}$ & 0.94 \\
\hline FGFR1 & $\begin{array}{l}\text { Chromosomal aberrations involving this gene are associated with stem cell myeloproliferative disorder and stem } \\
\text { cell leukemia lymphoma syndrome. }\end{array}$ & 0.94 \\
\hline IL2RG & The protein encoded by this gene is an important signaling component of many interleukin receptors & 0.92 \\
\hline ERBB2 & v-erb-b2 erythroblastic leukemia viral oncogene homolog 2, neuro/glioblastoma derived oncogene homolog & 0.92 \\
\hline FGFR3 & $\begin{array}{l}\text { This particular family member binds acidic and basic fibroblast growth hormone and plays a role in bone } \\
\text { development and maintenance. Mutations in this gene lead to craniosynostosis and multiple types of skeletal } \\
\text { dysplasia. }\end{array}$ & 0.9 \\
\hline AKT1 & v-akt murine thymoma viral oncogene homolog 1 & 0.9 \\
\hline INSR & insulin receptor & 0.9 \\
\hline IL2RA & Mutations in this gene are associated with interleukin 2 receptor alpha deficiency. & 0.9 \\
\hline SDC2 & $\begin{array}{l}\text { The syndecan- } 2 \text { protein functions as an integral membrane protein and participates in cell proliferation, cell } \\
\text { migration and cell-matrix interactions via its receptor for extracellular matrix proteins. Altered syndecan-2 expression } \\
\text { has been detected in several different tumor types. }\end{array}$ & 0.88 \\
\hline MAPK1 & $\begin{array}{l}\text { The protein encoded by this gene is a member of the MAP kinase family. MAP kinases, also known as extracellular } \\
\text { signal-regulated kinases (ERKs), act as an integration point for multiple biochemical signals, and are involved in a } \\
\text { wide variety of cellular processes such as proliferation, differentiation, transcription regulation and development. }\end{array}$ & 0.86 \\
\hline CD247 & $\begin{array}{l}\text { The protein encoded by this gene is T-cell receptor zeta, which together with T-cell receptor alpha/beta and } \\
\text { gamma/delta heterodimers, and with CD3-gamma, -delta and -epsilon, forms the T-cell receptor-CD3 complex. }\end{array}$ & 0.86 \\
\hline RET & ret proto-oncogene & 0.86 \\
\hline VEGFA & vascular endothelial growth factor $\mathrm{A}$ & 0.86 \\
\hline PTPN1 & protein tyrosine phosphatase, non-receptor type 1 & 0.86 \\
\hline IL3RA & The protein encoded by this gene is an interleukin 3 specific subunit of a heterodimeric cytokine receptor. & 0.84 \\
\hline HDAC1 & $\begin{array}{l}\text { histone deacetylase } 1 \text {, Together with metastasis-associated protein-2, it deacetylates p53 and modulates its effect } \\
\text { on cell growth and apoptosis. }\end{array}$ & 0.82 \\
\hline CCND1 & $\begin{array}{l}\text { The protein encoded by this gene belongs to the highly conserved cyclin family, whose members are characterized } \\
\text { by a dramatic periodicity in protein abundance throughout the cell cycle. This protein has been shown to interact } \\
\text { with tumor suppressor protein Rb and the expression of this gene is regulated positively by Rb. Mutations, } \\
\text { amplification and overexpression of this gene, which alters cell cycle progression, are observed frequently in a } \\
\text { variety of tumors and may contribute to tumorigenesis }\end{array}$ & 0.82 \\
\hline FASN & fatty acid synthase & 0.82 \\
\hline CD4 & $\begin{array}{l}\text { The protein functions to initiate or augment the early phase of T-cell activation, and may function as an important } \\
\text { mediator of indirect neuronal damage in infectious and immune-mediated diseases of the central nervous system. }\end{array}$ & 0.8 \\
\hline
\end{tabular}

Verified drug targets: Drug targets with the entry "Pharmacological action" labeled as 'Yes' in the Drugbank.

All 4013 GWAS reported genes and 1463 drug targets were mapped to NCBI gene IDs to provide unique identifiers for comparison. For the 88 GWAS diseases with drugs in Drugbank, there are 1914 GWAS reported genes and 821 drug targets. The verified drug target set has 353 genes for 81 diseases. For each disease, we compare the list of GWAS reported genes and drug targets and find the overlap between these two lists.

\section{Calculating expected overlap between GWAS reported genes and drug targets using a random model}

We assume there are 20,000 human genes. For a specific disease, if there are ' $m$ ' GWAS reported genes, and there are ' $n$ ' drug targets for this disease the expected random 
overlap between the two gene lists for that disease is $\mathrm{n} * \mathrm{~m} / 20000$. We calculated the expected overlap for each disease and summed these to get the expected total number of overlaps between drug targets and GWAS reported genes for the same disease.

SNP impact analysis for GWAS genes and drug target genes 1000 genomes VCF data were downloaded from http:// www.1000genomes.org/data. The 2010 November data set is used. We extracted all non-synonymous variants from 1000 genomes data based on Refseq annotation downloaded from the UCSC genome browser in Jan 2012, and calculated the allele frequency for each of the non-reference variants by dividing the number of alleles (count 1 for heterozygous and 2 for homozygous) by the number of total possible (2 times the number of people). We found non-synonymous SNPs in the coding regions of 3550 out of the 4013 GWAS reported genes and 1249 out of the 1463 drug targets.

The density of common non-synonymous SNPs in each gene is calculated by dividing the number of non-synonymous SNPs with frequencies $>5 \%$ for that gene by the length of that gene's protein sequence provided by the UCSC genome browser http://genome.ucsc.edu/. One splicing form is randomly chosen for each NCBI gene ID.

\section{Transcript length analysis}

The longest transcript for each drug target and GWAS reported gene was picked based on the Refseq annotation downloaded from the UCSC genome browser in Jan 2012.

\section{Evolutionary analysis for GWAS reported genes and Drug target genes}

Ratios of non-synonymous to synonymous substitution rates, $\mathrm{dN} / \mathrm{dS}$, for human proteins were downloaded from http://www.h-invitational.jp/evola/download.html in March 2012. The h-inv [34] IDs were converted to NCBI Gene IDs using a conversion map downloaded from http://biodb.jp/download.cgi. dN/dS from Human-Mouse orthologs and Human-Chimpanzee orthologs were selected. Human-Mouse $\mathrm{dN} / \mathrm{dS}$ are considered to reflect selection over a relatively long time period, and HumanChimpanzee $\mathrm{dN} / \mathrm{dS}$ to reflect more recent history.

\section{Human gene network analysis for GWAS reported genes and drug target genes}

The Functional Interaction protein network [21] was downloaded from http://genomebiology.com/content/supplementary/gb-2010-11-5-r53-s3.zip. This un-weighted map consists of 209,988 functional interactions involving 10956 proteins, and covers roughly half of the human coding genome. Gene symbols in this data set were converted to NCBI gene IDs. 1125 out of 1914 GWAS reported genes and 611 out of 821 drug target genes for the 88 diseases and 932 drug targets of all 1463 drug targets were mapped into the network.

The Floyd-Warshall algorithm [35] was used to calculate the shortest path between all gene pairs in the network. The resulting set of inter-node distances serves as a background distribution. For each disease, we extracted the set of all pairwise distances between GWAS genes for that disease, between drug targets genes, and between GWAS genes and drug target genes. For each disease, we also calculated the shortest path from every gene in the network to the nearest GWAS gene for that disease and to the nearest drug target for the disease.

\section{Machine learning for drug targets}

We used a random forest implemented in WEKA [27] to train on the $\mathrm{N}+3$ features to predict known drug targets for a disease from the set of all drug targets. The training sets are unbalanced since the number of drug targets for each disease is very small (median 28) compared to all possible drug targets, 932. We use the MetaCost procedure [36] to deal with the unbalanced training set, which gives more penalty to false negative errors than to false positive errors. We set the cost factor to be the ratio between the number of 'correct' and 'incorrect' drug targets. We set the parameter $\mathrm{K}$, the number of separating features, as the square root of the number of all features and set the parameter I, the number of decision trees in the random forest, as 50.10 fold cross validation was used to measure the performance for the random forest method for each disease.

\section{Discussion}

This work began with an evaluation of the capability of GWA studies to identify existing drug targets for complex trait disease, based on a comparison of proposed disease mechanism genes in the GWAS catalog and drug targets in Drugbank. To our surprise, only 20 of these 856 drug targets correspond to GWAS identified mechanism genes. Although the point is not emphasized there, a recent study also found a small level of overlap between GWAS disease genes and corresponding drug targets for approved drugs [37] (16 compared with our 20 , based on fewer GWAS genes, Table S3 in [37]). Interestingly, that study found that inclusion of targets for drugs at all stages of development boosts the overlap considerably, to 63. Thus it appears that drugs currently being developed are more commonly GWAS genes than those already approved, perhaps because new studies are now selecting targets from GWAS results. Another study has examined the possibility of repurposing based on overlap between OMIM disease genes and drug targets [38], and reports a higher level of overlap.

We investigated two possible reasons why the overlap of GWAS results and drug targets is so low. First, there 
may be more selection against SNPs with significant impact in drug targets. Studies [5] have shown that GWAS methods typically find high frequency SNPs with modest phenotype effects. On the other hand drug targets have big effect sizes with respect to disease phenotypes. Thus there may be fewer high frequency deleterious SNPs in these genes. Indeed, we do observe this trend for nonsynonymous SNPs through analysis of population genomics data from the 1000 genomes project. It is likely that SNPs exerting their influence through other mechanisms (for example, altering the regulation of the expression of genes, changing the splicing pattern, or changing the stability of messenger RNA) also follow the same pattern since selection pressure is independent of impact mechanism. This finding of apparent selection pressure against variants with impact on drug target activity is supported by the observation of similar trends in acceptance of species-specific changes, as measured through $\mathrm{dN} / \mathrm{dS}$.

The second possible reason why GWAS genes and drug target overlap is small that we investigated concerns the relative length of GWAS genes versus drug targets. We find that on average GWAS genes are very significantly longer than drug targets, by about a factor of two, and also longer than the set of all genes. These data suggest that mechanisms that are more likely to occur in longer transcripts, such as those involving missense SNPs, play a significant role in complex traits. The data do not rule out other explanations for the length differences, but in any case there is a strong length bias in GWAS genes.

These two factors - selection against common SNPs in drug targets and longer length of GWAS genes - are significant but may not be the only factors contributing to very low drug target/GWAS gene overlap. As discussed earlier, loss of overlap from data errors does not appear large, but incomplete coverage by typical microarrays is a contributing factor [3]. There are some other factors that will contribute. Drugs may act to alleviate symptoms rather than affect the disease itself or they may act in a more global non-specific manner, for example generally suppressing inflammation rather than influencing a specific disease. Also, drugs typically decrease the in vivo activity of the protein concerned, whereas altered activity of mechanism genes may affect disease traits through either a decrease or an increase of in vivo activity (for example, a SNP may result in up-regulation of expression, contributing to disease risk).

The fact that most existing drug targets are not rediscovered by GWAS does not necessarily imply that few new drug targets will be directly discovered through this technology. For example, many drug targets for inflammatory diseases provide general reduction of inflammation, while its possible that GWAS may lead to much more disease specific targets. What is clear is that the close relationship between drug targets and GWAS reported genes makes the GWAS genes valuable network reference points for finding new drug targets. We have shown that relatively simple machine learning methods are effective at identifying potential drug repurposing opportunities, and one of our initial short-listed repurposing candidates has now been approved for use by the FDA. There is clearly considerable scope for more sophisticated methods, employing a combination of network and pathway information.

The present GWAS technology is only able to detect disease associations involving common SNPs. There are a large number of rare variants in the human exome [39] and as exome sequence and full genome sequence replace DNA microarrays in GWAS studies [40], the role of these is becoming better defined. A deep re-sequencing project for drug target genes has found an abundance of rare functional variants [41] and these are likely to play a role in complex disease. For some diseases, such as hypertension, many candidate genes have been proposed using non-genomic methods [2]. Rare variants in these candidate genes in patients will also be of great interest.

\section{Abbreviations used}

Genome wide association study: GWAS; Single nucleotide variant: SNV; Single nucleotide polymorphism: SNP; Human Gene Mutation Database: HGMD; Support vector machine: SVM; Radial basis function: RBF.

\section{Additional material}

Additional file 1: Overlap between GWAS reported genes and validated drug targets

\section{Competing interests}

The authors declare they have no conflict of interests in relation to this SNPSIG issue article.

\section{Authors' contributions}

CC and JM conceived this work and participated in its design. CC performed all the analyses and machine learning. CC and JM wrote the manuscript. Both authors read and approved the manuscript.

\section{Acknowledgements}

We are grateful to Lipika Ray for many productive discussions, technical help, and generation of the list of all candidate genes in GWAS loci. This work was supported in part by NIH LM007174, GM102801, and GM104436.

\section{Declarations}

The publication costs for this article were funded by a grant from the National Institutes of Health GM104436.

This article has been published as part of BMC Genomics Volume 15 Supplement 4, 2014: SNP-SIG 2013: Identification and annotation of genetic variants in the context of structure, function, and disease. The full contents of the supplement are available online at http://www.biomedcentral.com/ bmcgenomics/supplements/15/S4 


\section{Authors' details}

${ }^{1}$ Institute for Bioscience and Biotechnology Research University of Maryland 9600 Gudelsky Drive, Rockville, MD 20850, USA. ${ }^{2}$ Computational Biology, Bioinformatics, and Genomics, Biological Sciences Graduate Program University of Maryland, College Park, MD 20742, USA. ${ }^{3}$ Department of Cell Biology and Molecular Genetics University of Maryland, College Park, MD 20742, USA.

Published: 20 May 2014

\section{References}

1. Corvin A, Craddock N, Sullivan P: Genome-wide association studies: a primer. Psychological medicine 2010, 40(7):1063.

2. Johnson AD, Newton-Cheh $C$, Chasman DI, Ehret GB, Johnson T, Rose L, Rice K, Verwoert GC, Launer LJ, Gudnason V, et al: Association of hypertension drug target genes with blood pressure and hypertension in 86,588 individuals. Hypertension 2011, 57(5):903-910.

3. Ehret GB: Genome-wide association studies: contribution of genomics to understanding blood pressure and essential hypertension. Curr Hypertens Rep 2010, 12(1):17-25.

4. Takahashi N, Smithies O: Human genetics, animal models and computer simulations for studying hypertension. Trends Genet 2004, 20(3):136-145.

5. Hindorff LA, Sethupathy P, Junkins HA, Ramos EM, Mehta JP, Collins FS, Manolio TA: Potential etiologic and functional implications of genomewide association loci for human diseases and traits. Proc Natl Acad Sci USA 2009, 106(23):9362-9367.

6. Knox C, Law V, Jewison T, Liu P, Ly S, Frolkis A, Pon A, Banco K, Mak C, Neveu V, et al: DrugBank 3.0: a comprehensive resource for 'omics' research on drugs. Nucleic Acids Res 2011, 39(Database):D1035-1041.

7. Overington JP, Al-Lazikani B, Hopkins AL: How many drug targets are there? Nature reviews Drug discovery 2006, 5(12):993-996.

8. Imming $P$, Sinning C, Meyer A: Drugs, their targets and the nature and number of drug targets. Nature reviews Drug discovery 2006, 5(10):821-834.

9. Durbin R, Altshuler D, Brooks L, Felsenfeld A, McEwen J: 1000 Genomes Project: A Deep Catalog of Human Genetic Variation. January; 2010

10. Stenson PD, Ball EV, Mort M, Phillips AD, Shiel JA, Thomas NS, Abeysinghe S, Krawczak M, Cooper DN: Human Gene Mutation Database (HGMD): 2003 update. Hum Mutat 2003, 21(6):577-581.

11. Kimura M: Preponderance of synonymous changes as evidence for the neutral theory of molecular evolution. Nature 1977, 267(5608):275-276.

12. Yamasaki C, Murakami K, Fujii Y, Sato Y, Harada E, Takeda J-i, Taniya T, Sakate R, Kikugawa S, Shimada M: The H-Invitational Database (H-InvDB), a comprehensive annotation resource for human genes and transcripts. Nucleic acids research 2008, 36(Database):D793.

13. Jia P, Zhao Z: Network-assisted Causal Gene Detection in Genome-wide Association Studies: An Improved Module Search Algorithm. IEEE Int Workshop Genomic Signal Process Stat 2011, 131-134.

14. Jia P, Wang L, Fanous AH, Pato CN, Edwards TL, Zhao Z: Network-assisted investigation of combined causal signals from genome-wide association studies in schizophrenia. PLoS Comput Biol; 2012:8(7):e1002587.

15. Yeh SH, Yeh HY, Soo WW: A network flow approach to predict drug targets from microarray data, disease genes and interactome network case study on prostate cancer. J Clin Bioinforma 2012, 2(1):1.

16. Ciriello G, Cerami E, Sander C, Schultz N: Mutual exclusivity analysis identifies oncogenic network modules. Genome Res 2012, 22(2):398-406.

17. Rossin EJ, Lage K, Raychaudhuri S, Xavier RJ, Tatar D, Benita Y, Cotsapas C, Daly MJ: Proteins encoded in genomic regions associated with immunemediated disease physically interact and suggest underlying biology. PLoS Genet 2011, 7(1):e1001273.

18. Breitkreutz BJ, Stark C, Reguly T, Boucher L, Breitkreutz A, Livstone M, Oughtred R, Lackner DH, Bahler J, Wood V, et al: The BioGRID Interaction Database: 2008 update. Nucleic Acids Res 2008, 36(Database):D637-640.

19. Chatr-aryamontri A, Ceol A, Palazzi LM, Nardelli G, Schneider MV, Castagnoli L, Cesareni G: MINT: the Molecular INTeraction database. Nucleic Acids Res 2007, 35:(Database issue):D572-574.

20. Kanehisa M, Goto S, Kawashima S, Okuno Y, Hattori M: The KEGG resource for deciphering the genome. Nucleic Acids Res 2004, 32:(Database issue): D277-280.

21. Wu G, Feng $X$, Stein $L$ : A human functional protein interaction network and its application to cancer data analysis. Genome Biol 2010, 11(5):R53.
22. Lee HK, Hsu AK, Sajdak J, Qin J, Pavlidis P: Coexpression analysis of human genes across many microarray data sets. Genome Res 2004 14(6):1085-1094

23. Prieto C, Risueno A, Fontanillo C, De las Rivas J: Human gene coexpression landscape: confident network derived from tissue transcriptomic profiles. PLoS One 2008, 3(12):e3911.

24. Verspoor K, Cohen KB, Lanfranchi A, Warner C, Johnson HL, Roeder C, Choi JD, Funk C, Malenkiy Y, Eckert M: A corpus of full-text journal articles is a robust evaluation tool for revealing differences in performance of biomedical natural language processing tools. BMC Bioinformatics 2012, 13(1):207.

25. Fire $M$, Tenenboim L, Lesser $\mathrm{O}$, Puzis $R$, Rokach L, Elovici Y: Link prediction in social networks using computationally efficient topological features. Privacy, security, risk and trust (passat), 2011 ieee third international conference on and 2011 ieee third international conference on social computing (socialcom): 2011 IEEE; 2011, 73-80.

26. Carley DW: Drug repurposing: identify, develop and commercialize new uses for existing or abandoned drugs. Part II. IDrugs: the investigational drugs journal 2005, 8(4):310

27. Witten $\mathbb{H}$, Frank E, Trigg LE, Hall MA, Holmes G, Cunningham SJ: Weka: Practical machine learning tools and techniques with Java implementations. 1999.

28. Korb LC, Ahearn JM: C1q binds directly and specifically to surface blebs of apoptotic human keratinocytes: complement deficiency and systemic lupus erythematosus revisited. The Journal of Immunology 1997 158(10):4525-4528.

29. Walport MJ, Davies KA, Botto M: C1q and systemic lupus erythematosus. Immunobiology 1998, 199(2):265-285.

30. Bowness P, Davies K, Norsworthy P, Athanassiou P, Taylor-Wiedeman J, Borysiewicz L, Meyer P, Walport M: Hereditary C1q deficiency and systemic lupus erythematosus. QJM 1994, 87(8):455-464.

31. Laxer R, Cameron B, Silverman E: Occurrence of Kawasaki disease and systemic lupus erythematosus in a single patient. The Journal of rheumatology 1988, 15(3):515.

32. Diniz J, Almeida R, Aikawa N, Sallum A, Sakane P, Silva C: Kawasaki disease and juvenile systemic lupus erythematosus. Lupus 2012, 21(1):89-92.

33. Gattei V, Degan M, Aldinucci D, De luliis A, Rossi FM, Mazzocco FT, Rupolo M, Zagonel V, Pinto A: Differential expression of the RET gene in human acute myeloid leukemia. Annals of hematology 1998, 77(5):207-210.

34. Imanishi T, Nakaoka H: Hyperlink Management System and ID Converter System: enabling maintenance-free hyperlinks among major biological databases. Nucleic Acids Res 2009, 37(Web Server):W17-22.

35. Floyd RW: Algorithm 97: shortest path. Communications of the ACM 1962 5(6):345.

36. Domingos P: MetaCost: a general method for making classifiers costsensitive. Proceedings of the fifth ACM SIGKDD international conference on Knowledge discovery and data mining: 1999 ACM; 1999, 155-164

37. Sanseau P, Agarwal P, Barnes MR, Pastinen T, Richards JB, Cardon LR, Mooser V: Use of genome-wide association studies for drug repositioning. Nature biotechnology 2012, 30(4):317-320.

38. Wang $Z Y$, Zhang $H Y$ : Rational drug repositioning by medical genetics. Nature biotechnology 2013, 31(12):1080-1082.

39. Tennessen JA, Bigham AW, O'Connor TD, Fu W, Kenny EE, Gravel S, McGee S, Do R, Liu X, Jun G, et al: Evolution and functional impact of rare coding variation from deep sequencing of human exomes. Science 2012, 337(6090):64-69.

40. Kiezun A, Garimella K, Do R, Stitziel NO, Neale BM, McLaren PJ, Gupta N, Sklar P, Sullivan PF, Moran JL: Exome sequencing and the genetic basis of complex traits. Nature genetics 2012, 44(6):623-630.

41. Nelson MR, Wegmann D, Ehm MG, Kessner D, Jean PS, Verzilli C, Shen J, Tang Z, Bacanu S-A, Fraser D: An abundance of rare functional variants in 202 drug target genes sequenced in 14,002 people. Science 2012, 337(6090):100-104.

doi:10.1186/1471-2164-15-S4-S5

Cite this article as: Cao and Moult: GWAS and drug targets. BMC Genomics 2014 15(Suppl 4):S5. 